\title{
Darfur: autodeterminación, colonialismo interno y separatismo etnopolítico en los primeros años del siglo XXI
}

\author{
Darfur: self-determinnation, domestic \\ colonialism and ethno-political secessionism \\ at the beginning of the 2lst century
}

\author{
Carlos Federico Domínguez Avila’
}

\section{Resumen}

l:1 articulo aborda la trayectorla det conflicto de larfur, asi como la coyumtura actual y algunos escenarios prospectivos. 11 argumentos contral del trabajo sugiere yue el conflicto emepolítion ỵu alli existe puede abordarse desde la opticia conceptual del colonialisme internos. Se concluye cue la alternativa más viahle palra las etmas fur, Masalit y Zaghawa -esto e's, los habitantes originales de Dartur- vendria a ser la recuperacion de su solerania politica, ecomomical social.

Palabras (anve: Darfur, Africa, comflictos conopoliticos, ecguridal internacional.

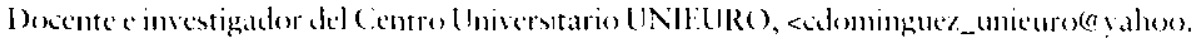
com.hr>

Recibido el 12 de sepriembre de 2008 ; aceptado d 10 de nowiembre de 2008. 
Abstract

The article approaches the trigectory of the contlict in Darfur, as well as the current compuncture and some prospective scenarios. 'The central argument suggests that the currene ethoupolitical conflict in l harfar can le examined from the conceptual standpome of internal colomialism. The paper conclude's that the most viable alternative to the Fur, Masalit and Zaghawa cthnias -original inhahitants of Darfar-would be the recovery of their political. economic and social sovercignty.

Ki-Worns: Darfur, Africa, ethnic and political conflicts, international security. 


\section{INTRODUCCIÓN}

Desde 2003 prevalece en la región de Darfur-oeste de Sudán- una de las mayores crisis humanitarias del mundo. Aunque existen ciertos acuerdos de cese al fuego, eventual reconciliación nacional y una modesta fuerza de paz integrada fundamentalmente por tropas africanas, lo cierto es que continúan llegando noticias de violencia etnopolítica desde la región.

Cicrtamente los peores momentos de la crisis de Darfur se produjeron entre febrero de 2003 y diciembre de 2004 (García, 2005). Ya pasó la época de las gravísimas matanzas, que incluso fueron consideradas como genocidio. Al parecer se redujo la violación masiva y organizada de los derechos humanos de las etnias Fur, Masalit y Zaghawa. Asimismo, mermó el saqueo, la destrucción y la quema indiscriminada de cosechas.

Con todo, en el segundo semestre de 2008 persiste el drama de Darfur. La mitad de los seis millones de habitantes originales de la región murieron ofueron forzados a emigrar hacia campos de refugiados en la frontera con Chad y la República Centroafricana o hacia otras ciudades del país -esto es, se convirtieron en desplazados internos para asegurar su propia supervivencia. La violencia paramilitar de las milicias progubernamentales -normalmente llamadas Janjaweed- produjo un peligroso vacío de poder político que favoreció la emergencia de la criminalidad organizada y común. Todo ello sin olvidar el trauma psicológico de una tragedia comparable a lo acontecido en Rwanda, Somalia, Bosnia, Afganistán o el Congo-por citar algunos de los casos más importantes de conflicto etnopolítico de los últimos años (Verschave, 2000; Straus, 200.5; Arrighi, 2002)1. Las grandes potencias con vínculos e intereses en el cuerno de África aparentemente no están dispuestas a detener definitivamente la tragedia. Y lo más probable es que en Darfur persistan el caos y la anarquía, a menos que la región consiga volver a ser independiente, como lo fue durante siglos -hasta su incorporación como dependencia del Imperio Británico, en 1917 (Held y otros, 1999).

Obsérvese que simultáneamente con la crisis en Darfur, las autoridades de Jartum y las guerrillas del Ejército de Liberación del Pueblo de Sudán -comandadas por John Garang-negociaban el llamado Acuerdo Amplio de Paz, firmado en 2005. Dicho acuerdo, aunque frágil e inestable, encerró formalmente la segunda guerra civil del país y abrió la posibilidad de realizar en 2011 un referendo popular con eventual separación e independencia del territorio. Al parecer, el éxito de la lucha etnopolítica y separatista de las comunidades del sur del país terminó motivando a los líderes de Darfur a lanzarse en una lucha semejante contra la desidia, el despotismo y el colonialis-

"De Rwaluda a Darfur", BBC, 6 de julio de 2007 , disponible en www blemundo. com, consultada el II de julio de 2008 . 
mo interno de las autoridades centrales sudanesas.

\section{LOS CONFLICTOS FINOPOLÍTICOS,} IA RLCOMPOSICJÓN DIE SISTIMA

WLSTFAI.IANO Y EL RESURGIMIENTO DE GULRRAS PRIEMOIDERNAS: BREVIS CONSIIDHRACIONI:S CONCLIPUAIIS

DISSIDE I A PERSPECIIVA DE IOS

I:SIUUIOS SOIBRE SE(FURIIAAI) INIERNACIONAL

A comienzos de la década de 1990 Francis Fukuyama se hizo famoso con su polémica hipótesis del fin de la historia. En términos fundamentales, el autor sostuvo que la mayoría de las naciones occidentales habrian entrado en una fase de estabilidad política y económica -quiere decir, el doble triunfo de la democracia representativa y del capitalismo liberal de mercado. De acuerdo con Fukuyama, el fin de la historia en occidente indicaba que no habia alternativas viables para aquellas naciones. Además, la mayoría de las naciones europeas y americanas habrian entrado en la fase de los conflictos posmodernos, cuya principal carncterística sería la reducida posibilidad de guerra o de violencia interestatal entre ellas. Con todo, el panorama propuesto por Fukuyama no se confirmó en muchos Fstados del mundo en desarrollo, donde una generación de conflictos armados intraestatales, básicamente identitarios o etnopolíticos, pasaron a ser preponderantes, violentos y altamente significativos en el escenario de la seguridad internacional (David, 2001). Tal fue el caso de Rwanda, Burundi, Bosnia y Chechenia, por citar algunos de los más conocidos.

Naturalmente los conflictos armados internos en general y los conflictos etnopolíticos en particular no son exclusivos del mundo pos Guerra Fría. Se trata de actos de violencia que predominaron hasta que surgió el llamado sistema westfaliano-basado en el principio de la soberanía y del Estado territorial (Krasner, 2001). En tal sentido, este tipo específico de conflicto se caracteriza por el predominio de la violencia civil, su naturaleza intraestatal y el predominio de rasgos identitarios socioculturales (especialmente de naturaleza religiosa, linguística, tribal o filosófica). A diferencia de una guerra interestatal en el sentido clásico del término, los conflictos armados vigentes en muchos países y regiones presentan una fuerte connotación intraestatal. La relevancia de la cuestión estrictamente territorial tiende a disminuir de manera apreciable como causa de la guerra. En general, el conflicto armado interno implica una situación en la cual un Estado se involucra en una oposición decidida contra una etnia, un clan o un grupo organizado, porque los objetivos procurados por unos y otros se hacen cada vez más incompatibles o antagónicos. En el caso de los conflictos etnopolíticos los objetivos son principalmente identitarios pero también políticos, diplomáticos, económicos y sociales'.

Vale reiterar que da mayoría de los contlic- 
Vale agregar que existen semejanzas y diferencias importantes entre los conflictos armados de naturaleza gucrrillera y separatista. En ambos casos el Estado pierde la adhesión y la legitimidad vertical-quiere decir, el pacto de autoridad, lealtad y sumisión. También son confliceros de larga duración y son particularmente violentos contra los no combatientes. Sin embargo, solo en el segundo caso se reivindicia la extinción de la solidaridad, cooperación y fraternidad horizontales, esto es, el pacto de unión perpetua entre los integrantes de una sociedad nacional debido a la emergencia de los micro-nacionalismos,

tos armados internos se desarrollan en paises africanes, astatices y del (Oriente Medio. 1)ada la naturaleza intraestatal de tales actos de violemeil generalmente no existen combates entere ejercitos organizados y eyuivalentes. fin consecterledia, los soldades entran carda vez menos én guerras. Sin tmbargo, los civiles son carda vez. más importantess sa como victimas a comocombatientes. Fllo acontece compuntamente con la urbanizacion de las guterras, el resurgimiento de mercenarios y de milicias tribales, la dramática situacion de los asi llamados "niños-soldados" y la desinstitucionalizacion de la guerra, centre otros tenomenos semejantes (David, 2(0)1).

Aproximadamente 300 endias de terdes les continentes podrian revindicar an listade propionen las proximas decadas amparandose en el principio de la autodeterminación de los pueblos. Se trata de naciones sin listiado propio. lillo incluye a los kurdos, palcestinos, vascor, chereseses, chechenios, tamile's, sicilianos, yuebequense's, entre muchos otros. 1 a independencia de tales pueblos se justitica especialmente cuando som olipeto de volencia masival fo al coliapso de la identidad nacional, a la desidia de las autoridades, a la incapacidad de ofrecer protección para garantizar la supervivencia, al despotismo y a la desintegración de Fstados corródes por la opresión y la corrupción ${ }^{+}$. F.n consccuencia, la lógica de los conflictes identitarios o etmopolíticos es particularmente atroz, dado que normalmente se pide la eliminación física de los "otros" para garantizar "muestra" supervivencia y valores.

Algunos autores llaman a este fenómeno retribalización o neomedievalismo. Una rejvindicación de la cultura local-tribal ante un sistema estatal wesfaliano que muestra evidentes signos de agotamiento en todo el mundo. Tal es el caso específico de ciertos países africanos herederos de modelos y sistemas políticos transplantados por los colonizadores europeos y que aparentemente no han podido -y dificilmente lograrán-constituir sociedades nacionales realmente democráticas, libres

genocidios por parte de las antoridades centralles de los respectivos paises.

+ A manera de cjemplo de contlictom armades internos cabe mencionar lon ciases de (o)lombia (guerrilla) y Filipinas (separa-

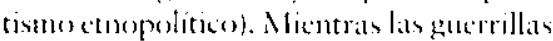
(1) AR(), los paramilitares (Alle.). los narcotraticantes y el ejercitecolombianos lachan dentro de las fronteras internaciomalnente reconocidas del pais - stn reivindicar mingun tipo de separatismo -, enc el archipielage assictice cohabitan fuet/als guterilleras, sparatistas, paramilitares y propianente estatales, con la posible secesion del sur del pais, en la hipotesis de uta victoria etmopelitica del Frente More de Liluracion Istamical y el Alu Saygat. 
Y justas. Al parecer, la mentalidad del colonizador europeo se reprodujo en las prácticas de las elites gobernantes dando lugar, entre otras cosas, a un virtual colonialismo interno aún vigente y preponderante en muchas naciones multiétnicias.

Subyacente a lo anterior y fundamental en el resto del presente articulo es el concepto de seguridad humana -propio de las modernas concepciones de seguridad internacional. Este concepto reivindica la proteción estatal y en ciertos casos supranacional de los derechos humanos fundamentales, particularmente cuando existe el riesgo de violaciones masivas y eventual genocidio de minorías en función de criterios socioculturales o de colonialismo interno. Se sostiene que en determinadas y críticas circunstancias, el principio de la soberanía estatal no dehe ser obstáculo para actuar en favor de las vítimas-ejemplo de lo cual son las intervenciones humanitarias. Se entiende que ciertos Fstados pueden terminar siendo una amenaza a la supervivencia de comunidades enteras de seres humanos, sea por su despotismoo por su desidia. En tales circunstancias, el derecho y la política internacionales han confirmado la relevancia de los criterios de la responsabilidad de pro. teger $y$ del derecho/deber de injerencia. lin comsecuencia, el Fstado deja de ser el único protector reconocido como legítimo de su sociedad. En algumos casos pasa a ser considerado una amenaza vital y puede ser cuestionado en lo concerniente a sus asuntos internos.
Así, en casos extremos de despotismo o desidia estatales se perfila la posibilidad de una acción concertada de la comunidad internacional en favor de las víctimas de violaciones masivas de los derechos humanos (David, 2001; Laceras, 2008).

\section{TRAGEdia E INDIFERENCIA EN DARlUUR: AIGUNOS ANTECIIDINTES DEL CONFIICTO}

Los antecedentes históricos del conflicto de Darfur son bastante antiguos y consistentes. Para los fines del presente artículo basta destacar que hasta su incorporación en la colonia británica de Sudán, la etnia Fur fue soherana y autónoma (Wesseling, 1998). Se trataba fundamentalmente de un reino sedentario dedicado a actividades agricolas. Con su independencia, en 19.56, el poder politico del inmenso país fue asumido por la elite política árabe de la region centro-norte. Nótese que desde antes de la independencia sudanesa la contradicción entre los árabes del valle del Nilo y etnias minoritarias no-írabes del sur, del oeste y del este fue clara y altamente significiativa (O'Fahey, 2006; Verschave, 2000).

Dicha contradicción resultó en dos guerras civiles sucesivas en el sentido Norte-Sur: 1955-1972 y 1983-2005, además del conflicto en Darfur desde 2003. En otras palabras, descle su independencia el país solo tuvo diez años de paz debido a las persistentes divergencias etnopolíticas entre la tradicional- 
mente dominante comunidad instalada en Jartum y algunas comunidades noárabes de la periferia sudanesa. Fn tal sentido, en Darfur es fundamental la cuestión centro-periferia o, expresado en términos más sociológicos, la cuestión del colonialismo interno. Se parte aquí de la constatación de que el gobierno de Jartum pretende mantener su hegemonía político-económica al mismo tiempo que las fuerzas etnopolíticas del interior procuran conseguir crecientes capacidades de autodeterminación y eventual independencia. En el caso específico de Darfur hay que añadir la vital cuestión de la supervivencia de las etnias Fur, Masalit y Zaghawa (Prendergast y Thomas-Jensen, 2007).

Conviene agregar que el actual gobierno de Sudán es presidido por el general Omar al-Bashir. Se trata de un gobierno autoritario resultado del golpe militar de 1989. Al comienzo, al-Bashir se inspiró ideológicamente en el fundamentalismo islámico, llegando incluso a acoger en el país al propio Osama bin Laden-lo que provocó gran preocupación en países occidentales y resultó en un bombardeo estadounidense a Jartum en 1998. La aproximación de al-Bashir at Partido del Congreso Nacional, surgido del Frente Nacional Istámico y dirigido durante muchos años por Hassan al-Turabi, permitió crear una base política de sustentación más o menos permanente y significativa para el gobierno, especialmente en la región centro-norte del país. Fn los últimos años al-Bashir ha abandonado el discurso mesianico en favor de un creciente pragmatismo y de una gradual aproximación a Washington y Beijing. Fn el campo económico, la creciente exploración de yacimientos petrolíteros permitió que Jartum financiara a los aliados gubernamentales, así como al aparato de seguridad del Estado -en desmedrode los intereses prioritarios de desarrollo humano equilibrado del país.

Muchos autores informan que el detonante directo del conflicto en Darfur fue justamente el malestar de los residentes de la región ante el abandono y la desidia de las autoridades centrales de Jartum. Así, en febrero de 2003, dos pequeños grupos insurgentes -denominados Ejército de I.jheración de Sudán (SLA) y Movimiento para la Justicia y la Igualdad (JEM) - atacaron puestos policiales y militares en el territorio de Darfur. La respuesta de Jartum consistió en aplicar una estrategia contrainsurgente de naturaleza paramilitar (y de bajo costo político y financiero), muy semejante a la empleada anteriormente en el sur del país. Ello derivó en la creación de milicias autorizadas para realizar una verdadera campaña de tierra arrasada en la región de Darfur, que culminó con la tragedia de los años 2003 y 2004 y cuyas consecuencias persisten hasta hoy. Obsérvese que, para Jartum, los acontecimientos en Darfur también encuadran en una lucha contra fuerzas separatistas que amenazan la unidad, la soberanía y la propia supervivencia del Estado sudanés (Krasner. 2001; Walzer, 20011).

Conviene agregar que los problemas etno- 
Desde 2004 se ha procurado llegar a acuerdos de pacificación y reconciliación entre el gohierno de al-Bashir y las numerosas denominaciones rebeldes de Darfur. En algunos casos estos intentos fueron respaldados por mediadores internacionales (Naciones Unidas, Unión Africana, gobierno de Libia). Entre estos acuerdos cabe destacar el alto al fuego de 2004, el Acuerdo de Paz de Darfur de 2006 y los esfuerzos de mediación de la Unión Africana y de las Naciones Unidas, que culminaron en el envío de una Misión de Paz africana en Darfur (2007-2008). Naturalmente, estas iniciativas son loables y han contribuido de forma más o menos eficiente a reducir las hostilidades, la violencia y la muerte de inocentes (Alaminos, $2008)^{h}$.

Entretanto, las causas profundas del conflicto en Darfur continúan vigentes. Aunque corriendo el riesgo de parecer simplista, parece evidente que muchos de los principales problemas de esa región en particular y de Sudán en general, se relacionan con: (a) el aparentemente incluctable proceso de

políticos existen -en diterente grado de intensidad- en casi todos los países del mundo, y particularmente en el mundo atroasiático. Obsérvese que ni en la propia Furopa (Ocidental el modelo de Fistado nacional iulealizado por Hohles y sucesores consiguió imponerse plenamente $y$ entrenta significativos desafíos en los primeros años del siglo xiy (Creveld, $2(10) 4)$.

6 ".isionon: poner fin a guerras en Africa", BBC:, 2.6.2008, disponilile en: www hhe: mundo.com, consultada of 11 de fulio de 2008. desintegración del Estado sudanés; (b) los conflictos centro-periferia (o de colonialismo interno) que desde la época de la independencia han plagado las relaciones entre la elite gobernante y comunidades no-árabes del oeste, sur y este del immenso país; (c) el acceso a recursos naturales (agua, tierra fértil, petróleo), que es vital para estudiar los problemas vigentes en Darfur y en el resto del país; (d) los problemas de gobernabilidad, la explotación y la desidia de las autoridades centrales en un país cuyo gobierno es claramente despótico, $y(e)$ la influencia y pretensiones de potencias externas con vínculos e intereses divergentes en Darfur y en Sudán.

En relación con los actores exógenos vale hacer una breve referencia vinculada a las actividades de potencias mundiales y regionales, así como a actores no estatales (instituciones religiosas y humanitarias, grupos de traficantes de armas, corporaciones, organizaciones separatistas del sur de Sudán que apoyan a los rebeldes de Darfur, entre otros). En pocas palabras, los Estados Unidos tienen intereses de

Desde 1984 la región de Darfur enfrenta un agudo proceso de desertificación y prolongida sequía que no ha dejado de influir en las crecientes divergencias $y$ contradicciones cntre los agricultores sedentarios de las etnias fur, Masalit y Zaghawa, de un lado, y nómades "árabes", (Abdalla, 2006). Este último grupo terminó siendo reclutado, armado y apoyado por el gobjerno central de Suclán, resultando en las llamadas milicias Janjaweed, grupo paramilitar responsable de las peores arrocidades del conflicto. 
seguridad en Sudán en el marco global de su guerra contra el terrorismo y en Washington también tienen alguna resonancia presiones ejercidas por grupos humanitarios y religiosos internos (Chomsky, 2007). China tiene crecientes intereses petroleros en Sudán -en consecuencia, Beijing tiende a apoyar y a mostrarse tolerante con el gobierno de al-Bashir (García, 2006). Rusia y Francia venden armamentos a Jartum. Fgipto, Etiopía, Arabia Saudita y en menor medida Eritrea trabajan para mantener la integridad territorial de Sudán e impulsar a al-Bashir a adoptar una opción moderada o pragmática. El gobierno de Chad tiende a apoyar a ciertas facciones rebeldes de Darfur y del sur de Sudán, lo que ha provocado graves tensiones y una virtual guerra no declarada con Jartum ${ }^{8}$.

\section{DARFur en La aCtuAlidad: EL PRECIO DE LA AUTODETERMINACIÓN}

Aun reconociendo los problemas de credibilidad de las estadísticas sobre victimas asociadas a situaciones tan dramáticas como la de Darfur, parece importante señalar que el conflicto ha

\footnotetext{
"Darfur incendia Africa central", El Pais, 21.6.2008, disponible en: www.elpais. com, consultada el 1.5 de julio de 2008 . Conviene agregar que existen vínculos de naturalezal tribal entre el presidente de Chad, latriss I éby, y algunas facciones de rebeldes de Darfur, yue tambien luchan contra el gobierno de al-Bashir.
}

provocado la muerte de no menos de 300 mil personas (el gobierno de Sudán reconoce hasta 9 mil victimas)". Lo que sucede es que instituciones como las Naciones Unidas y especialmente las organizaciones no gubernamentales que trabajan en el terreno tienen en cuenta tanto los asesinados directamente como las víctimas del hambre, de la penuria y de la desolación que sucedió a la lucha armada. Asimismo, unas $200 \mathrm{mil}$ personas de Darfur se concentran en campos de refugiados localizados en las proximidades de las fronteras con Chad y la República Centroafricana. Unos 3 millones de personas afectadas por el conflicto precisan ayuda internacional para sobrevivir dentro del propios Darfur. Un número indeterminado de desplazados internos procuró refugio en otros lugares de Sudán. En pocas palabras, prácticamente la mitad de los seis millones de habitantes originales de Darfur fue asesinada o sobrevive lejos de su tierra de origen (Natsios, 2008) ${ }^{14}$.

A los gravísimos problemas humanitarios citados debe agregarse el virtual vacío de poder político predominante en la región. Se trata de una virtual "somalización" de Darfur en el sentido de que las autoridades sudanesas no tienen el control efectivo de la violencia 
en la región. Ello genera una situación de virtual retroceso al estado de naturaleza -en el sentido hobbesiano del término.

Los rebeldes de Darfur sufren un agudo proceso de fragmentación a ráz. del cual han surgido casi 30 minúsculas organizaciones etnopolíticas dirigidas por caudillos, jefes tribales y señores de la guerra que tienen variedad de intereses y exigencias. El vacio de poder, la falta de seguridad y el caos reinante en las zonas lejanas de Darfur favorece la difusion de la criminalidad, del paramilitarismo y del bandolerismo común. La falta de unidad de acción y la polifonía insurgente dificulta mucho el proceso de pacificación del país debido a la dificultad para conseguir acuerdos políticos de validez general. En consecuencia, los acuerdos parciales alcanzados suelen ser extremadamente volátiles -y casi siempre terminan siendo ignorados por ambos lados. Una eventual democratización de Darfur y de Sudán simplemente no existe como ohjetivo de mediano o largo plazo.

Asimismo, la elite militar de Sudán se aferra decididamente al poder. Aparentemente al-Bashir y todo el aparato gubernament: l vigente en el país entienden que no lun.trian futuro en caso de scr climinados. Dan por sentado que, si son desalojados del poder, se verían amenazados por venganzas y purgas endógenas y por posibles juicios por crímenes contra la humanidad en cl escenario internacional. En consecuencia, al-Bashir está dispuesto a luchar utilizando el argumento nacionalista del mantenimiento y resguardo de la soheranía y de la integridad territorial del país. Como segunda alternativa, al parecer estaría dispuesto a aceptar la separación de regiones periféricas o desprovistas de recursos naturales fundamentales (agua, petróleo, tierras fértiles), aunque conservando el poder en la vital región centro-norte del país.

Ejemplo de esta virtual balcanización de Sudán es la tensa coexistencia existente desde la firma del Acuerdo Amplio de Paz (en 2005) entre el gobierno de Jartum y los rebeldes del sur de Sudán -que no incluye la situación existente en Darfur, aunque termina enlazándose directa e indirectamente con ella. En última instancia, el acuerdo prevé la realización de un referendo en 2011 para definir la posible (y plausible) separación del sur de Sudán y el nacimiento de un nuevo país con capital en Juba. Al parecer, la elite gobernante de Jartum estaría dispuesta a aceptar la eventual independencia del sur del país. Sin embargo, ello no incluye a Darfur (oeste) ni a otra región separatista del este del país. No está claro si cl gobierno central de Sudán estaría dispuesto a continuar luchando para mantener su predominio en territorios de población no-árabe, lo que ciertamente implica costos humanos y económicos muy altos -inclusive para un país con crecientes ingresos provenientes de la exportación de petróleo". Paralelamente, el ejemplo

1 Desde 1998 Sudáu se crigió en exportador de petroileo, principalmente hacia cl mercado asiático (China, India, Malasia). La creciente renta petrolera permite cooptar oponentes, getuerar prosperidad para la 
y el precedente de los pucblos del sur de Sudán, que tras años de guerra civil (1983-2005) consiguieron un amplio grado de autodeterminación y buenas perspectivas de independencia definitiva, puede ser de interés para los pueblos Fur, Masalit y Zaghawa, especialmente en un contexto de gravísimas disputas interétnicas como las que predominan en el inmenso Sudán ${ }^{12}$.

\section{CONSIDERACIONES FINALES:} FSCENARIOS PROSPECIIVOS PARA DARFUR (2008-201. I)

Darfur y Sudán continuarán enfrentando en los próximos años enormes desafíos y disyuntivas políticas, económicas, sociales y de seguridad.

clite del valle det Nilo y consolidar el aparato de seguridad del Fstado. Los petrodólares también ayudan a reducir el impacto político de la presión externa en asuntos espinoses, como el de Darfur. Las relaciones sino-sudanesis son particularmente importante en este sentido, dado cue Beijing ofrece a Jartum el así llamado "pacquete completo"-quiere decir, apoyo político en el escenario internacional, ayuda económica y rransferencia de antals- a cambio de altas y crecicutes exportaciones de petróleo sudanés hacial el pais asiótico (K/cincAhlbrandt y Small, 2008; (iancia, 2006).

1: Darfur corre el riesgo de una "somalización» no solamente en el sentido de predominio de una situación de caos, anarquía e indefinición. También existe el riesgo de caer en la indiferencia, la banalización y la irrelevancia bajo la perspectiva de la comunidad internacionat. "Dartur: einditerencia estrepitosa?", BBC, 23.4.2008, disponible cn www.bbemundocom, consultata el 11 de julio de 2008.
Lamentablemente los escenarios más plausibles no son favorables o halagüeños ${ }^{\prime 3}$. Salvo mejor interpretación de las tendencias actualmente existentes, es previsible y razonable proponer dos escenarios principales y dos escenarios alternativos.

El primer escenario es incrcial y básicamente implica la continuidad de las tendencias observadas en los últimos meses. En la práctica, sugiere una virtual «somalización" de Darfur y con el tiempo, de todo el país. Se trata de una situación de caos, anarquía y total ausencia de poder político común -con todas las consecuencias implicitas, particularmente en lo concerniente a delincuencia, bandolerismo y desintegración sociopolítica. Para algunos autores se trata de una situación de Estado fallido o de simple regresión al estado de naturaleza -en el pesimista sentido hobbesiano del término (Creveld, 2004) $)^{1 \cdot}$.

1" "litenviadode la ONll a Sudáncuestiona la voluntad para alcanzar la pazs, fifF, 24.6.2008, disponible en: www.espanol. news yahoo.com, consultada el 15 de julio de 2008 .

14 Con esas ideas en mente, conviene agregar que varios autores recomocen el agotamiento del lestado territorial y la emergencia de formas, estructuras y modelos de organización política generados a partir de realidades lociales y también en el contextode las transformaciones globales. tin el caso especifico de los paises africanos se trata de sistemas sociales, politicos y economicos contrastantes con el modele de Estado nacional de origen turopere e impuesto desde el exterior (Krasner, 200); Kobrin, 1998). 
Un scgundo escenario principal sería la separación e independencia de Darfur respecto de Sudán. En otras palabras, se trata de seguir el ejemplo de los acuerdos alcanzados entre Jartum y los pueblos del sur del país -que permiten visualizar una independencia más o menos pacífica del territorio en 2011. La propia teoría realista recomienda que en casos de balcanización irreversible es mejor permitir la independencia de las unidades periféricas, preservando la región central de los países. Las nuevas naciones estarían sujetas a las presiones y a la lógica del sistema de Estados. En tal sentido, Darfur formaría parte de la conocida tendencia hacia la simultánea tragmentación, desintegración y proliferación de países (Creveld, 2004). Con todo, es difícil dar por hecha la viabilidad funcional de un país destrozado por la guerra, con un territorio de casi 500 mil kilómetros cuadrados y una población traumatizada de 5 millones de hab.....es -fundamentalmente de las etnıci i ur, Masalit y Zaghawa. Es posible que antes de una independencia desordenada e inconsecuente haya que considerar la posibilidad de una intervención humanitaria que permita constituir una institucionalidad básica -de orientación democrática y popular.

Un tercer escenario, menos plausible que los anteriores, sería la reanudación de los enfrentamientos armados tanto en Darfur como en el sur de Sudán, como consecuencia de la negativa de Jartum a aceptar los separatismos periféricos. la recomposición de la estabilidad autoritaria es una alterna- tiva plausible en caso de que el ejército local logre sofocar por la fuerza los movimientos secesionistas. Otra hipótesis podria ser resultado de la derrota militar de la tradicional elite dominante en el país y su substitución por representantes de los otrora pueblos periféricos. La tradicionalmente dominante elite sudanesa sería substituida y al mismo tiempo se garantizaría la integridad territorial del país. Esto es, habría una "africanización» del hasta ahora predominantemente arabizado Sudán. No está claro si ello implicaría una verdadera solución de los graves problemas políticos del país o si, por el contrario, sería una nueva fase del conflicto etnopolítico imperante en el país. Lo más probable es que, en caso de sobrevivir, el Estado sudanés se transforme en una entidad vegetativa -esto es, un Estado desprovisto de monopolio sobre la violencia o sobre el mantenimiento del orden jurídico, o que simplemente dejó de funcionar comotal ${ }^{15}$.

Finalmente, el escenario menos plausible propone la simultánea pacificación, reconciliación y democratiza-

Es evidente que el modelo de Fstado nacional surgio en Europa Occidental con los trabajos de l lobbes y sucesores. Ia transposicion del modelo a orros continentes fue muy difícil. Normalmente el modelo de Estado nacional ha tenido yue coexistir y cohahitar con formas premodernas de organización política. En consecuencia, seguramente los pucblos continuarán su propio rumbo, dispensando e ignorando autoridades sin legitimidad, $y$ en un mundo con Fstados en declinacion. 
ción del país. Naturalmente, muchos podrían considerar que esta sería la mejor alternativa para mantener la unidad nacional, la soberanía, la integridad territorial y la convivencia libre y justa de todos los habitantes del país. Sin embargo, la constitución de un Estado multiétnico y pluricultural es prácticamente inviable en un país con las actuales características de Sudán debido a los persistentes problemas de tribalismo y baja adhesión a una identidad nacional específica en el país.

A juicio del autor de este artículo, la realidad político-militar de Darfur y de Sudán terminará avanzando por algún punto intermedio entre el segundo y el tercero de los escenarios planteados. En otras palabras, se trataría de la constitución de un Darfur con amplia autonomía para administrar sus asuntos internos y en un plazo razonable lograr la independencia completa y definitiva respecto de Sudán.

\section{Posdata de agosto de 2008}

El 14 de julio de 2008, Luis Moreno Ocampo, fiscal jefe del Tribunal Penal Internacional (TPI), con sede en La Haya, Holanda, solicitó a los jueces de dicha corte que emitieran una orden internacional de arresto contra el presidente al-Bashir y el congelamiento de sus cuentas bancarias. El mandatario sudanés es acusado de crimenes de guerra y de lesa humanidad en relación con los dramáticos acontecimientos ocurrridos en Darfur desde 2003. Aunque el gobier- no de Sudán no reconoce la competencia jurídica del Tribunal Penal Internacional, sus fiscales iniciaron sus actividades de acuerdo con el mandato otorgado por la resolución 1593 del Consejo de Seguridad de las Naciones Unidas el 31 de marzo de 2005. No está claro si los jucces admitirán las pruelas presentadas por Moreno Ocampo para encausar al presidente al-Bashir, inclusive porque es la primera vez que un mandatario en funciones es acusado y responsabilizado de crímenes contra la humanidad. Como mínimo, la acusación sugiere que en Darfur siguen produciéndose los acontecimientos y que los dictadores no pueden impunemente continuar cometiendo actos de genocidio ${ }^{1 t}$.

\section{REFERENCIAS BIBLIOGRÁFICAS}

Ahdalla, Abadia(2006), "Environmental Degradation and Conflict in Darfur: Experience and Development (Options"., Entironmental Degradation as al Cattse of Conflict in Darfur; Jartum, Naciones Utidias,pp.. 87-94.

Alaminos, Naría Śngeles (20)8), "lil conflicto de Darfur: un reto para la credibilidad de la Unión Africana", UNISCI Discussion Papers, n. 16, pp. 229-253.

in "Fl TPI pide la detencion del presidente de Suctán por la tragedia de Darfur", El Pais, 14.7.2008, disponible en: www.elpais.com, consultada el 15 de julio de 2008. "Fiscal de (.1'l pide inculpación! orden de arresto contra presidente sudanés", AFP, 14.7.2008, disponible e'n: www.espanol.news.yalhoo.com, consultada cl $1.5 \mathrm{de}$ julio de 2008 . 
Arriglu, (iovamni. "The African (risis/ World Systemic and Regional Aspects". Now left Reviene, n. 15, 2002, pp. 5-36.

(homsky, Noam (2007), Fstades fallidos/ Fl abuso de poder y el ataque a la democracia. Barcelona: Fdiciones B.

(reveld, Martin van (2004), Ascensän é declino do Histado, São laulo, Martins Fontes.

David, Charles-l'hilippe (2001), A Guera a a Pazl Alordagens contemporâneas da sogmanca e da estratégia, I.isboa, Piaget.

Crarcía, Carlota (2006), "La política africana de Pekín: eoportunidad o amenaza?", Analisis de Real Instituto Elcano, ARI 27-2006, Madrid,: Real Instituto Ekcano, disponible en <www.realinstitutoclcano. org>, consultada cl II de julio de 2008 .

- "La Comunidad Internacional y Darfur" (2005), Análisis de' Real Instituto Plamo, ARI 62-2005, Mladrict, Real Instituto Ekano, disponible en <www: realinstitutoelcano.org>, consultada ol 11 de julio de 2008 .

Held, Davidy otros (1999), Transformaciones globales/ Politica, economia y' cultura, Cundad de México, Oxford..

Kleine-Ahllorandt, Stephanic y Andrew Small (2008), "La nueva diplomacia de China hacia las dictaduras", Foreign Affairs ('n Fspañol, (abril-junio), disponible en <www.foreignaffairs-esp.org>, comsultada (l) 11 de julio de 2008 .

Kobrin. Stephen J. (1999), "Back to the Fature: Neomedievalism and the Postmodern Digital World Economy", The University of P'tunsylvania, 1999, disponible en www-management. whartom.upenn.edu/
krobrin/Research/hartrev2.pdf, consultada el 9 de septiembre de 2008 .

Krasner, Stephen D. (2001), Solerania, hipocresia organizada. Barcelona, Paiclós,

1.aceras, Borja. (2008), "Darfur y la Responsabilidad de lroteger", Politica Exterior n". 124, pp. 107-118.

Natsios, Andrew. (02008), "Beyond Darfur/ Sudan's Slide Toward Civil War". Foreign Affairs, v. 87, n". 3, pp. 77-93.

()'Fahey, R.S. (2006), "Conflict In I)arfur Historical and Contemporary Perspectives", Enimonmental Degradation as a Canse of Confliat in Darfur. Jartum, Naciones Unidas, pp. 2.3-32.

P'rendergast, John y Colin Thomas-Jensen (2007), "F.l Gran Cuerno de Africa: cambiar de política", Foretgn Affairs en Espanol (julio-septiembre), disponible en <www. foreignaffairs-esp.org >, consultada d 11 de julio de 2008 .

Straus, Scout (2006), "Darfur and the Gonocide Debate", Foreign dffairs, (January-Fehruary), disponihle en < www. forcignaffairsorg $>$, comsultada el II de julio de 2008 .

Versschateve, Françnis-Xavier (200)0), Noir siluncel Qui arrêtera la Françafrique? Paris, Les Arènes.

Walter, Nichacl (2001), Cinerras justase injustas/ In razonantento moral con ejemplos bistóricos, Barcelona, Paidós.

Wesscling, H.I. (1998), Ditidir para dominaw A partilha da Africa 1880-1914, Rio de Janeiro, Felitora UFRJ, Revan. 